\title{
Positive periodic solutions for third-order ordinary differential equations with delay
}

He Yang ${ }^{1 *}$ (D) and Yujia Chen ${ }^{1}$

\section{${ }^{*}$ Correspondence:}

yanghe256@163.com

${ }^{1}$ College of Mathematics and

Statistics, Northwest Normal

University, Lanzhou, People's

Republic of China

\begin{abstract}
This paper deals with the existence of positive $\omega$-periodic solutions for third-order ordinary differential equation with delay

$$
u^{\prime \prime \prime}(t)+M u(t)=f(t, u(t), u(t-\tau)), \quad t \in \mathbb{R},
$$

where $\omega>0$ and $M>0$ are constants, $f: \mathbb{R}^{3} \rightarrow \mathbb{R}$ is continuous, $f(t, x, y)$ is $\omega$-periodic in $t$, and $\tau>0$ is a constant denoting the time delay. We show the existence of positive $\omega$-periodic solutions when $0<M<\left(\frac{2 \pi}{\sqrt{3} \omega}\right)^{3}$ and $f$ satisfies some order conditions. The discussion is based on the theory of fixed point index.
\end{abstract}

MSC: 34B18; 34C25

Keywords: Third-order differential equation; Positive $\omega$-periodic solution; Positive cone; Delay; Fixed point index theory in cones

\section{Introduction}

In this paper, we discuss the existence of positive $\omega$-periodic solutions for the third-order ordinary differential equation with delay

$$
u^{\prime \prime \prime}(t)+M u(t)=f(t, u(t), u(t-\tau)), \quad t \in \mathbb{R}
$$

where $M>0$ is a constant, $f: \mathbb{R}^{3} \rightarrow \mathbb{R}$ is continuous, $f(t, x, y)$ is $\omega$-periodic in $t, \tau>0$ is a constant which denotes the time delay.

The problem of periodic solutions for delayed differential equations is an important research topic in ODE qualitative analysis, which has wide applications in mechanics, physics, ecology, economics, and other disciplines and has attracted much attention of scholars; see [3, 5-12, 14]. For second-order differential equations without delay, the existence and multiplicity of positive periodic solutions are discussed in [1, 5-10, 12, 14]. In [13], the authors studied the existence of positive solutions for higher order $p$-Laplacian boundary value problems. In recent years, the existence of positive periodic solutions for third-order ODEs has been studied by using fixed point theorems of cone mapping. By utilizing Krasnoselskii's fixed point theorem in cones Feng [3] proved the existence and multiplicity of positive periodic solutions of the third-order equation

$$
u^{\prime \prime \prime}(t)+\alpha u^{\prime \prime}(t)+\beta u^{\prime}(t)=f(t, u(t)), \quad t \in[0,2 \pi]
$$

(c) The Author(s) 2018. This article is distributed under the terms of the Creative Commons Attribution 4.0 International License (http://creativecommons.org/licenses/by/4.0/), which permits unrestricted use, distribution, and reproduction in any medium, provided you give appropriate credit to the original author(s) and the source, provide a link to the Creative Commons license, and indicate if changes were made. 
where $\alpha>0$ and $\beta>0$ satisfy certain conditions. $\operatorname{Li}[11]$ discussed the existence of positive $\omega$-periodic solutions for the fully third-order ODE

$$
u^{\prime \prime \prime}(t)=f\left(t, u(t), u^{\prime}(t), u^{\prime \prime}(t)\right), \quad t \in \mathbb{R}
$$

By applying the fixed point theorem of cone expansion or compression type, the author established some existence results of positive $\omega$-periodic solutions of Eq. (1.3). However, all these works contain no delay terms, and few researchers consider the existence of positive periodic solutions for the third-order delayed Eq. (1.1). In this paper, we show the existence of positive $\omega$-periodic solutions for the third-order delayed Eq. (1.1) when $0<M<\left(\frac{2 \pi}{\sqrt{3} \omega}\right)^{3}$ and $f$ satisfies some order conditions. The discussion is based on the theory of fixed point index.

The rest of this paper is organized as follows. In Sect. 2, we introduce some preliminary facts and establish the existence of $\omega$-periodic solution for third-order linear differential equation with delay. In Sect. 3, we prove two existence theorems of positive $\omega$-periodic solutions for the third-order delayed Eq. (1.1).

\section{Preliminaries}

Let $C_{\omega}(\mathbb{R})$ denote the Banach space of all continuous $\omega$-periodic function $u(t)$ with norm $\|u\|_{C}=\max _{0 \leq t \leq \omega}|u(t)|$. We denote by $C_{\omega}^{+}(\mathbb{R})$ the cone of positive functions in $C_{\omega}(\mathbb{R})$.

Letting $M>0$, we first consider the linear third-order boundary value problem (BVP)

$$
\left\{\begin{array}{l}
u^{\prime \prime \prime}(t)+M u(t)=0, \quad t \in[0, \omega] \\
u^{(i)}(0)=u^{(i)}(\omega), \quad i=0,1, \\
u^{\prime \prime}(0)=u^{\prime \prime}(\omega)+1
\end{array}\right.
$$

By Lemma 2.1 of [1] the BVP (2.1) has a unique solution. The solution $\Psi(t)$ of BVP (2.1) has the following property (see Lemma 2.2 of [11] for details).

Lemma 1 ([11], Lemma 2.2) Let $0<M<\left(\frac{2 \pi}{\sqrt{3} \omega}\right)^{3}$. Then the solution $\Psi$ of the $B V P(2.1)$ is positive on $[0, \omega]$.

Secondly, for any $h \in C_{\omega}(\mathbb{R})$, we consider the existence of an $\omega$-periodic solution of the linear third-order ordinary differential equation

$$
u^{\prime \prime \prime}(t)+M u(t)=h(t), \quad t \in \mathbb{R} .
$$

For Eq. (2.2), we have the following lemma.

Lemma 2 ([11], Lemma 2.1) Let $M>0$. Then for any $h \in C_{\omega}(\mathbb{R})$, the linear Eq. (2.2) has a unique $\omega$-periodic solution $u(t)$ expressed by

$$
u(t)=\int_{t-\omega}^{t} \Psi(t-s) h(s) d s:=(P h)(t), \quad t \in \mathbb{R}
$$

Moreover, $P: C_{\omega}(\mathbb{R}) \rightarrow C_{\omega}(\mathbb{R})$ is a completely continuous linear operator. 
Remark 1 By Lemma $1, \Psi(t)>0$ for every $t \in[0, \omega]$ when $0<M<\left(\frac{2 \pi}{\sqrt{3} \omega}\right)^{3}$. Combining this fact with Lemma 2 we have that $P: C_{\omega}(\mathbb{R}) \rightarrow C_{\omega}(\mathbb{R})$ is a positive operator when $0<M<$ $\left(\frac{2 \pi}{\sqrt{3} \omega}\right)^{3}$.

Now, let $M>0$ and $M_{1}>0$. For any $h \in C_{\omega}(\mathbb{R})$, we consider the existence of an $\omega$-periodic solution of the linear third-order ordinary differential equation

$$
u^{\prime \prime \prime}(t)+M u(t)+M_{1} u(t-\tau)=h(t), \quad t \in \mathbb{R} .
$$

Lemma 3 Let $0<M<\left(\frac{2 \pi}{\sqrt{3} \omega}\right)^{3}$ and $0<M_{1}<M$. Then Eq. (2.4) has a unique $\omega$-periodic solution $u \in C_{\omega}(\mathbb{R})$ given by

$$
u(t)=\left(I+P \circ B_{1}\right)^{-1} \int_{t-\omega}^{t} \Psi(t-s) h(s) d s, \quad t \in \mathbb{R},
$$

where $B_{1}: C_{\omega}(\mathbb{R}) \rightarrow C_{\omega}(\mathbb{R})$ is defined by

$$
B_{1} u(t)=M_{1} u(t-\tau)
$$

Proof By the definition of $B_{1}$, it is easy to see that $B_{1}$ is a linear bounded operator and $\left\|B_{1}\right\| \leq M_{1}$. Equation (2.4) is equivalent to the equation

$$
u^{\prime \prime \prime}(t)+M u(t)=h(t)-B_{1} u(t), \quad t \in \mathbb{R}
$$

By Lemma 2, Eq. (2.6) has a unique $\omega$-periodic solution given by

$$
\begin{aligned}
u(t) & =\int_{t-\omega}^{t} \Psi(t-s)\left[h(s)-B_{1} u(s)\right] d s \\
& =\int_{t-\omega}^{t} \Psi(t-s) h(s) d s-\int_{t-\omega}^{t} \Psi(t-s) B_{1} u(s) d s
\end{aligned}
$$

From this equation we obtain

$$
\left(I+P \circ B_{1}\right) u(t)=\int_{t-\omega}^{t} \Psi(t-s) h(s) d s, \quad t \in \mathbb{R} .
$$

By (2.3), for any $h \in C_{\omega}(\mathbb{R})$, we have

$$
\begin{aligned}
|(P h)(t)| & \leq \int_{t-\omega}^{t}|\Psi(t-s)| \cdot|h(s)| d s \\
& \leq \int_{t-\omega}^{t} \Psi(t-s) d s \cdot\|h\|_{C} \\
& =\int_{0}^{\omega} \Psi(t) d t \cdot\|h\|_{C} \\
& =\frac{1}{M}\|h\|_{C} .
\end{aligned}
$$

This implies that

$$
\|P\| \leq \frac{1}{M}
$$


Hence $\left\|P \circ B_{1}\right\| \leq\|P\| \cdot\left\|B_{1}\right\| \leq \frac{M_{1}}{M}<1$. So, $\left(I+P \circ B_{1}\right)^{-1}$ exists, and

$$
\left\|\left(I+P \circ B_{1}\right)^{-1}\right\| \leq \frac{1}{1-\left\|P \circ B_{1}\right\|} \leq \frac{M}{M-M_{1}} .
$$

Hence from (2.7) we have

$$
u(t)=\left(I+P \circ B_{1}\right)^{-1} \int_{t-\omega}^{t} \Psi(t-s) h(s) d s, \quad t \in \mathbb{R},
$$

which is the unique $\omega$-periodic solution of Eq. (2.4). This completes the proof of Lemma 3.

By Lemma 1, if $0<M<\left(\frac{2 \pi}{\sqrt{3} \omega}\right)^{3}$, then the solution of Eq. (2.1) $\Psi(t)>0$ for every $t \in[0, \omega]$. In this case, let $\Psi^{*}=\max _{t \in[0, \omega]} \Psi(t), \Psi_{*}=\min _{t \in[0, \omega]} \Psi(t)$, and $\sigma=\frac{\Psi_{*}}{\Psi^{*}}$; then $0<\sigma<1$. Define the operator $Q: C_{\omega}(\mathbb{R}) \rightarrow C_{\omega}(\mathbb{R})$ by

$$
(Q h)(t)=\left(I+P \circ B_{1}\right)^{-1} \int_{t-\omega}^{t} \Psi(t-s) h(s) d s, \quad t \in \mathbb{R} .
$$

We first prove that $Q$ is a positive operator.

Lemma 4 Let $0<M<\left(\frac{2 \pi}{\sqrt{3} \omega}\right)^{3}$ and $0<M_{1}<\sigma^{2} M$. Then $Q: C_{\omega}(\mathbb{R}) \rightarrow C_{\omega}(\mathbb{R})$ is a positive operator, where $Q$ is defined by (2.8).

Proof By Lemma 2 and Remark 1, for any $h \in C_{\omega}^{+}(\mathbb{R})$, we have $(P h)(t) \geq \sigma(P h)(s)$ for $t, s \in \mathbb{R}$. Particularly,

$$
(P h)(t) \geq \sigma \varepsilon_{0}, \quad(P h)(t) \leq \frac{1}{\sigma} \varepsilon_{0}, \quad \forall t \in \mathbb{R},
$$

where $\varepsilon_{0}=(P h)(0) \geq 0$ is regarded as a constant. Since

$$
\left(I+P \circ B_{1}\right)^{-1}=\sum_{i=0}^{\infty}(-1)^{i}\left(P \circ B_{1}\right)^{i}=\sum_{i=0}^{\infty}\left(P \circ B_{1}\right)^{2 i}\left(I-P \circ B_{1}\right),
$$

by (2.8) we only need to prove that $\left(I-P \circ B_{1}\right) P$ is positive. In fact, for any $h \in C_{\omega}^{+}(\mathbb{R})$, we have

$$
\begin{aligned}
(I & \left.-P \circ B_{1}\right)(P h)(t) \\
& =(P h)(t)-\left(P \circ B_{1}\right)(P h)(t) \\
& \geq \sigma \varepsilon_{0}-\frac{1}{\sigma}\left(P \circ B_{1}\right) \varepsilon_{0} \\
& \geq \sigma \varepsilon_{0}-\frac{M_{1}}{\sigma M} \varepsilon_{0} \\
& =\left(\sigma-\frac{M_{1}}{\sigma M}\right) \varepsilon_{0} \geq 0, \quad \forall t \in \mathbb{R} .
\end{aligned}
$$

This implies that $\left(I-P \circ B_{1}\right) P$ is positive. Therefore $Q: C_{\omega}(\mathbb{R}) \rightarrow C_{\omega}(\mathbb{R})$ is a positive operator. This completes the proof of Lemma 4. 
Choose the cone $K$ in $C_{\omega}^{+}(\mathbb{R})$ by

$$
K=\left\{u \in C_{\omega}^{+}(\mathbb{R}): u(t) \geq \sigma u(s), \forall t, s \in \mathbb{R}\right\}
$$

Then we have the following lemma.

Lemma 5 Let $0<M<\left(\frac{2 \pi}{\sqrt{3} \omega}\right)^{3}$ and $0<M_{1}<\sigma^{2} M$. Then $Q: K \rightarrow K$ is completely continuous, where $Q$ is defined by (2.8).

Proof For any $h \in K$, by (2.8) we have

$$
(Q h)(t)=\left(I+P \circ B_{1}\right)^{-1} \int_{t-\omega}^{t} \Psi(t-s) h(s) d s, \quad t \in \mathbb{R},
$$

namely,

$$
\left(I+P \circ B_{1}\right)(Q h)(t)=\int_{t-\omega}^{t} \Psi(t-s) h(s) d s, \quad t \in \mathbb{R},
$$

which implies

$$
\left(I+P \circ B_{1}\right)(Q h)(s) \leq \Psi^{*} \int_{s-\omega}^{s} h(\theta) d \theta=\Psi^{*} \int_{0}^{\omega} h(\theta) d \theta .
$$

On the other hand, for any $t \in \mathbb{R}$, we have

$$
\left(I+P \circ B_{1}\right)(Q h)(t) \geq \Psi_{*} \int_{t-\omega}^{t} h(\theta) d \theta=\Psi_{*} \int_{0}^{\omega} h(\theta) d \theta .
$$

From the two inequalities it follows that

$$
\left(I+P \circ B_{1}\right)(Q h)(t) \geq \sigma\left(I+P \circ B_{1}\right)(Q h)(s), \quad \forall t, s \in \mathbb{R} .
$$

By Lemmas 2 and 4 it is easy to see that $\left(I+P \circ B_{1}\right)^{-1}$ is a bounded positive operator. Hence $Q: K \rightarrow K$ is completely continuous. This completes the proof of Lemma 5 .

Applying the fixed point index theory in cones to prove the existence of $\omega$-periodic solutions of Eq. (1.1), we recall some concepts and conclusions on the fixed point index in $[2,4]$. Let $E$ be a Banach space, and let $K \subset E$ be a closed convex cone in $E$. Assume that $\Omega$ is a bounded open subset of $E$ with boundary $\partial \Omega$ and $K \cap \partial \Omega=\theta$, where $\theta$ denotes the zero element in $E$. Let $A: K \cap \bar{\Omega} \rightarrow K$ be a completely continuous mapping. If $A u \neq u$ for any $u \in K \cap \partial \Omega$, then the fixed point index $i(A, K \cap \Omega, K)$ is defined. If $i(A, K \cap \Omega, K) \neq 0$, then $A$ has a fixed point in $K \cap \Omega$. The following lemmas can be found in [4].

Lemma 6 Let $\Omega$ be a bounded open subset of $E$ with $\theta \in \Omega$, and let $A: K \cap \bar{\Omega} \rightarrow K$ be a completely continuous mapping. If

$$
\lambda A u \neq u, \quad u \in K \cap \partial \Omega, 0<\lambda \leq 1,
$$

then $i(A, K \cap \Omega, K)=1$. 
Lemma 7 Let $\Omega$ be a bounded open subset of $E$, and let $A: K \cap \bar{\Omega} \rightarrow K$ be a completely continuous mapping. If there exists $e \in K \backslash\{\theta\}$ such that

$$
u-A u \neq \mu e, \quad \forall u \in K \cap \partial \Omega, \mu \geq 0,
$$

then $i(A, K \cap \Omega, K)=0$.

\section{Existence of positive periodic solutions}

Theorem 1 Let $f(t, x, y): \mathbb{R} \times \mathbb{R}^{+} \times \mathbb{R}^{+} \rightarrow \mathbb{R}^{+}$be continuous and $\omega$-periodic in $t$. Suppose that $0<M<\left(\frac{2 \pi}{\sqrt{3} \omega}\right)^{3}$ and $f$ satisfies the following conditions:

$\left(\mathrm{H}_{1}\right)$ there exist $a_{1}>0$ and $a_{2}>0$ with $a_{1}+a_{2}<M$ and $\delta>0$ such that

$$
f(t, x, y) \leq a_{1} x+a_{2} y
$$

for any $t \in \mathbb{R}$ and $x, y \in[0, \delta]$;

$\left(\mathrm{H}_{2}\right)$ there exist $b_{1}>0$ and $b_{2}>0$ with $b_{1}+b_{2}>M$ and $h_{0} \in C_{\omega}^{+}(\mathbb{R})$ such that

$$
f(t, x, y) \geq b_{1} x+b_{2} y-h_{0}(t)
$$

for any $t \in \mathbb{R}$ and $x, y \in \mathbb{R}^{+}$.

Then Eq. (1.1) has at least one positive $\omega$-periodic solution.

Proof Let $M_{1} \geq 0$. Equation (1.1) is equivalent to the equation

$$
u^{\prime \prime \prime}(t)+M u(t)+M_{1} u(t-\tau)=f(t, u(t), u(t-\tau))+M_{1} u(t-\tau), \quad t \in \mathbb{R} .
$$

Let $F(u)(t)=f(t, u(t), u(t-\tau))+M_{1} u(t-\tau)$. Then by condition $\left(\mathrm{H}_{1}\right) F: C_{\omega}^{+}(\mathbb{R}) \rightarrow C_{\omega}^{+}(\mathbb{R})$ is bounded. If $0<M_{1}<\sigma^{2} M$, then by Lemma 3 and 5 we get that $A=Q \circ F: C_{\omega}^{+}(\mathbb{R}) \rightarrow C_{\omega}^{+}(\mathbb{R})$ is completely continuous, where $Q$ is defined by (2.8). For any $0<r<R<+\infty$, let

$$
\Omega_{r}=\left\{u \in C_{\omega}^{+}(\mathbb{R}):\|u\|_{C}<r\right\}, \quad \Omega_{R}=\left\{u \in C_{\omega}^{+}(\mathbb{R}):\|u\|_{C}<R\right\}
$$

Clearly, $\Omega_{r}$ and $\Omega_{R}$ are bounded open subsets of $C_{\omega}^{+}(\mathbb{R})$. We show that $A$ has a fixed point in $K \cap\left(\Omega_{R} \backslash \bar{\Omega}_{r}\right)$ when $r$ is small enough and $R$ is large enough.

Let $r \in(0, \delta)$. We prove that $\lambda A u \neq u$ for any $u \in K \cap \partial \Omega_{r}$ and $0<\lambda \leq 1$, where $K$ is defined by (2.9). In fact, if there exist $u_{0} \in K \cap \partial \Omega_{r}$ and $0<\lambda_{0} \leq 1$ such that

$$
\lambda_{0} A u_{0}=u_{0}
$$

then

$$
u_{0}^{\prime \prime \prime}(t)+M u_{0}(t)+M_{1} u_{0}(t-\tau)=\lambda_{0} F\left(u_{0}\right)(t), \quad t \in \mathbb{R},
$$

namely,

$$
u_{0}^{\prime \prime \prime}(t)+M u_{0}(t) \leq f\left(t, u_{0}(t), u_{0}(t-\tau)\right), \quad t \in \mathbb{R} .
$$


Since $u_{0} \in K \cap \partial \Omega_{r}$, it follows that

$$
u_{0}(t) \leq\left\|u_{0}\right\|_{C}=r<\delta, \quad u_{0}(t-\tau) \leq\left\|u_{0}\right\|_{C}=r<\delta .
$$

Hence by condition $\left(\mathrm{H}_{1}\right)$ we have

$$
u_{0}^{\prime \prime \prime}(t)+M u_{0}(t) \leq a_{1} u_{0}(t)+a_{2} u_{0}(t-\tau), \quad t \in \mathbb{R}
$$

Integrating both sides of this inequality from 0 to $\omega$ and using the periodicity of $u_{0}$, we have

$$
\begin{aligned}
M \int_{0}^{\omega} u_{0}(t) d t & \leq a_{1} \int_{0}^{\omega} u_{0}(t) d t+a_{2} \int_{0}^{\omega} u_{0}(t-\tau) d t \\
& =\left(a_{1}+a_{2}\right) \int_{0}^{\omega} u_{0}(t) d t
\end{aligned}
$$

Since $u_{0} \in K$, it follows that $u_{0}(t) \geq \sigma u_{0}(s)$ for any $t, s \in \mathbb{R}$. Hence

$$
\left(M-a_{1}-a_{2}\right) \sigma \omega u_{0}(s) \leq\left(M-a_{1}-a_{2}\right) \int_{0}^{\omega} u_{0}(t) d t \leq 0, \quad \forall s \in \mathbb{R}
$$

Since $M>a_{1}+a_{2}, \sigma>0$, and $\omega>0$, (3.1) implies that $u_{0}(s) \leq 0$ for any $s \in \mathbb{R}$, which contracts to $u_{0} \in K \cap \partial \Omega_{r}$. Hence $A$ satisfies the conditions of Lemma 6. By Lemma 6 we have

$$
i\left(A, K \cap \Omega_{r}, K\right)=1 .
$$

On the other hand, let $e(t) \equiv 1$ for any $t \in \mathbb{R}$. Then $e \in K \backslash\{\theta\}$. We show that $u-A u \neq \mu e$ for any $u \in K \cap \partial \Omega_{R}$ and $\mu \geq 0$ when $R$ is large enough. In fact, if there exist $u_{1} \in K \cap \partial \Omega_{R}$ and $\mu_{1} \geq 0$ such that

$$
u_{1}-A u_{1}=\mu_{1} e
$$

then $u_{1}-\mu_{1} e=A u_{1}$. Hence

$$
u_{1}^{\prime \prime \prime}(t)+M u_{1}(t)-\mu_{1}\left(M+M_{1}\right)=f\left(t, u_{1}(t), u_{1}(t-\tau)\right), \quad t \in \mathbb{R}
$$

By condition $\left(\mathrm{H}_{2}\right)$ we have

$$
u_{1}^{\prime \prime \prime}(t)+M u_{1}(t) \geq b_{1} u_{1}(t)+b_{2} u_{1}(t-\tau)-h_{0}(t), \quad t \in \mathbb{R}
$$

Integrating both sides of this inequality from 0 to $\omega$ and using the periodicity of $u_{1}$, we have

$$
\begin{aligned}
M \int_{0}^{\omega} u_{1}(t) d t & \geq b_{1} \int_{0}^{\omega} u_{1}(t) d t+b_{2} \int_{0}^{\omega} u_{1}(t-\tau) d t-\int_{0}^{\omega} h_{0}(t) d t \\
& =\left(b_{1}+b_{2}\right) \int_{0}^{\omega} u_{1}(t) d t-\int_{0}^{\omega} h_{0}(t) d t
\end{aligned}
$$


namely,

$$
\left(b_{1}+b_{2}-M\right) \int_{0}^{\omega} u_{1}(t) d t \leq \int_{0}^{\omega} h_{0}(t) d t \leq \omega\left\|h_{0}\right\|_{C} .
$$

Since $u_{1} \in K$ and $u_{1}(t) \geq \sigma u_{1}(s)$ for any $t, s \in \mathbb{R}$, we have

$$
\left(b_{1}+b_{2}-M\right) \omega \sigma u_{1}(s) \leq \omega\left\|h_{0}\right\|_{C},
$$

namely,

$$
\left\|u_{1}\right\|_{C} \leq \frac{1}{\sigma\left(b_{1}+b_{2}-M\right)}\left\|h_{0}\right\|_{C}:=\bar{R} .
$$

Let $R>\max \{r, \bar{R}\}$. Then $A$ satisfies the conditions of Lemma 7 in $\Omega_{R}$. By Lemma 7 we have

$$
i\left(A, K \cap \Omega_{R}, K\right)=0 .
$$

Combining (3.2) with (3.3), we have

$$
i\left(A, K \cap\left(\Omega_{R} \backslash \bar{\Omega}_{r}\right), K\right)=i\left(A, K \cap \Omega_{R}, K\right)-i\left(A, K \cap \Omega_{r}, K\right)=-1 \neq 0 .
$$

Hence $A$ has at least one fixed point in $K \cap\left(\Omega_{R} \backslash \bar{\Omega}_{r}\right)$, which is a positive $\omega$-periodic solution of Eq. (1.1). This completes the proof of Theorem 1.

Theorem 2 Let $f(t, x, y): \mathbb{R} \times \mathbb{R}^{+} \times \mathbb{R}^{+} \rightarrow \mathbb{R}^{+}$be continuous and $\omega$-periodic in $t$. Suppose that $0<M<\left(\frac{2 \pi}{\sqrt{3} \omega}\right)^{3}$ and $f$ satisfies the following conditions:

$\left(\mathrm{H}_{3}\right)$ there exist $b_{1}>0$ and $b_{2}>0$ with $b_{1}+b_{2}>M$ and $\delta>0$ such that

$$
f(t, x, y) \geq b_{1} x+b_{2} y
$$

for any $t \in \mathbb{R}$ and $x, y \in[0, \delta]$;

$\left(\mathrm{H}_{4}\right)$ there exist $a_{1}>0$ and $a_{2}>0$ with $a_{1}+a_{2}<M$ and $h_{1} \in C_{\omega}^{+}(\mathbb{R})$ such that

$$
f(t, x, y) \leq a_{1} x+a_{2} y+h_{1}(t)
$$

for any $t \in \mathbb{R}$ and $x, y \in \mathbb{R}^{+}$.

Then Eq. (1.1) has at least one positive $\omega$-periodic solution.

Proof Let $F(u)(t)=f(t, u(t), u(t-\tau))+M_{1} u(t-\tau)$, and $A=Q \circ F$. Then $A: C_{\omega}^{+}(\mathbb{R}) \rightarrow C_{\omega}^{+}(\mathbb{R})$ is completely continuous when $0 \leq M_{1}<\sigma^{2} M$. For any $0<r<R<+\infty$, we prove that $A$ has a fixed point in $K \cap\left(\Omega_{R} \backslash \bar{\Omega}_{r}\right)$ when $r$ is small enough and $R$ is large enough.

Let $r \in(0, \delta)$, and let $e(t) \equiv 1$ for any $t \in \mathbb{R}$. Then $e \in K \backslash\{\theta\}$. If there exist $u_{0} \in K \cap \partial \Omega_{r}$ and $\mu_{0} \geq 0$ such that $u_{0}-A u_{0}=\mu_{0} e$, namely, $u_{0}-\mu_{0} e=A u_{0}$, then by the definition of $A$ and Lemma $3 u_{0}$ satisfies

$$
u_{0}^{\prime \prime \prime}(t)+M u_{0}(t)-\mu_{0}\left(M+M_{1}\right)=f\left(t, u_{0}(t), u_{0}(t-\tau)\right), \quad t \in \mathbb{R},
$$


that is,

$$
u_{0}^{\prime \prime \prime}(t)+M u_{0}(t) \geq f\left(t, u_{0}(t), u_{0}(t-\tau)\right), \quad t \in \mathbb{R} .
$$

Since $u_{0} \in K \cap \partial \Omega_{r}$, it follows that

$$
u_{0}(t) \leq\left\|u_{0}\right\|_{C}=r<\delta, \quad u_{0}(t-\tau) \leq\left\|u_{0}\right\|_{C}=r<\delta .
$$

Hence by condition $\left(\mathrm{H}_{3}\right)$ we have

$$
u_{0}^{\prime \prime \prime}(t)+M u_{0}(t) \geq b_{1} u_{0}(t)+b_{2} u_{0}(t-\tau), \quad t \in \mathbb{R} .
$$

Integrating both sides of this inequality from 0 to $\omega$ and using the periodicity of $u_{0}$, we have

$$
\begin{aligned}
M \int_{0}^{\omega} u_{0}(t) d t & \geq b_{1} \int_{0}^{\omega} u_{0}(t) d t+b_{2} \int_{0}^{\omega} u_{0}(t-\tau) d t \\
& =\left(b_{1}+b_{2}\right) \int_{0}^{\omega} u_{0}(t) d t .
\end{aligned}
$$

Since $u_{0} \in K$, it follows that $u_{0}(t) \geq \sigma u_{0}(s)$ for any $t, s \in \mathbb{R}$. Hence

$$
\left(M-b_{1}-b_{2}\right) \int_{0}^{\omega} u_{0}(t) d t \geq\left(M-b_{1}-b_{2}\right) \sigma \omega u_{0}(s) \geq 0, \quad \forall s \in \mathbb{R} .
$$

Since $M<b_{1}+b_{2}, \sigma>0$, and $\omega>0$, (3.4) implies that $u_{0}(s) \leq 0$ for any $s \in \mathbb{R}$, which contracts to $u_{0} \in K \cap \partial \Omega_{r}$. Hence $A$ satisfies the conditions of Lemma 7. By Lemma 7 we have

$$
i\left(A, K \cap \Omega_{r}, K\right)=0 .
$$

On the other hand, we show that $A$ satisfies the condition of Lemma 6 in $K \cap \Omega_{R}$ when $R$ is large enough. In fact, if there exist $u_{1} \in K \cap \partial \Omega_{R}$ and $0<\lambda_{1} \leq 1$ such that

$$
\lambda_{1} A u_{1}=u_{1}
$$

then we have

$$
u_{1}^{\prime \prime \prime}(t)+M u_{1}(t)+M_{1} u_{1}(t-\tau)=\lambda_{1} F\left(u_{1}\right)(t), \quad t \in \mathbb{R} .
$$

By condition $\left(\mathrm{H}_{4}\right)$ we have

$$
u_{1}^{\prime \prime \prime}(t)+M u_{1}(t) \leq a_{1} u_{1}(t)+a_{2} u_{1}(t-\tau)+h_{1}(t), \quad t \in \mathbb{R} .
$$

Integrating both sides of this inequality from 0 to $\omega$ and using the periodicity of $u_{1}$, we have

$$
\begin{aligned}
M \int_{0}^{\omega} u_{1}(t) d t & \leq a_{1} \int_{0}^{\omega} u_{1}(t) d t+a_{2} \int_{0}^{\omega} u_{1}(t-\tau) d t+\int_{0}^{\omega} h_{1}(t) d t \\
& =\left(a_{1}+a_{2}\right) \int_{0}^{\omega} u_{1}(t) d t+\int_{0}^{\omega} h_{1}(t) d t,
\end{aligned}
$$


that is,

$$
\left(M-a_{1}-a_{2}\right) \int_{0}^{\omega} u_{1}(t) d t \leq \int_{0}^{\omega} h_{1}(t) d t \leq \omega\left\|h_{1}\right\|_{C} .
$$

Since $u_{1} \in K, u_{1}(t) \geq \sigma u_{1}(s)$ for any $t, s \in \mathbb{R}$, we have

$$
\left(M-a_{1}-a_{2}\right) \omega \sigma u_{1}(s) \leq \omega\left\|h_{1}\right\|_{C}
$$

that is,

$$
\left\|u_{1}\right\|_{C} \leq \frac{1}{\sigma\left(M-a_{1}-a_{2}\right)}\left\|h_{1}\right\|_{C}:=R^{*} .
$$

Let $R>\max \left\{r, R^{*}\right\}$. Then $A$ satisfies the conditions of Lemma 6 . By Lemma 6 we have

$$
i\left(A, K \cap \Omega_{R}, K\right)=1 .
$$

Combining (3.5) with (3.6), we have

$$
i\left(A, K \cap\left(\Omega_{R} \backslash \bar{\Omega}_{r}\right), K\right)=i\left(A, K \cap \Omega_{R}, K\right)-i\left(A, K \cap \Omega_{r}, K\right)=1 \neq 0 .
$$

Hence $A$ has at least one fixed point in $K \cap\left(\Omega_{R} \backslash \bar{\Omega}_{r}\right)$, which is a positive $\omega$-periodic solution of Eq. (1.1). This completes the proof of Theorem 2.

\section{Conclusion}

In this paper, by utilizing the fixed point index in cones, we prove the existence of positive periodic solutions for the general third-order Eq. (1.1). The results are obtained in the case that $f$ satisfies some order conditions. A similar method can be used to prove the existence of positive periodic solutions for other differential equations.

Funding

The research is supported by the National Natural Science Function of China (No. 11701457) and Gansu Technology Plan (No. 17JR5RA071).

\section{Competing interests}

None of the authors has any competing interests in the manuscript.

Authors' contributions

Both authors contributed equally in writing this paper. Both authors read and approved the final manuscript.

\section{Publisher's Note}

Springer Nature remains neutral with regard to jurisdictional claims in published maps and institutional affiliations.

Received: 21 May 2018 Accepted: 23 July 2018 Published online: 07 August 2018

\footnotetext{
References

1. Cabada, A.: The method of lower and upper solutions for second, third, fourth and higher order boundary value problem. J. Math. Anal. Appl. 185, 302-320 (1994)

2. Deimling, K.: Nonlinear Functional Analysis. Springer, New York (1985)

3. Feng, Y: On the existence and multiplicity of positive periodic solutions of a nonlinear third-order equation. Appl. Math. Lett. 22, 1220-1224 (2009)

4. Guo, D.J., Lakshmikantham, V:: Nonlinear Problems in Abstract Cones. Academic Press, New York (1988)

5. Jiang, D.Q., Nieto, J., Zuo, W.J.: On monotone method for first and second-order periodic boundary value problems and periodic solutions of functional differential equations. J. Math. Anal. Appl. 289, 691-699 (2004)
} 
6. Jiang, D.Q., Wei, J.J.: Monotone method for first-and second-order periodic boundary value problems and periodic solutions of functional differential equations. Nonlinear Anal. 50, 885-898 (2002)

7. Li, Q., Li, Y.X.: On the existence of positive periodic solutions for second-order functional differential equations with multiple delays. Abstr. Appl. Anal. 2012, Article ID 929870 (2012)

8. Li, Q., Li, Y.X.: Existence and multiplicity of positive periodic solutions for second-order functional differential equations with infinite delay. Electron. J. Differ. Equ. 2014, 93 (2014)

9. Li, Y.X.: Positive periodic solutions of nonlinear second order ordinary differential equations. Acta Math. Sin. 45, 481-488 (2002) (in Chinese)

10. Li, Y.X.: Positive periodic solutions of first and second order ordinary differential equations. Chin. Ann. Math., Ser. B 25, 413-420 (2004)

11. Li, Y.X.: Positive periodic solutions for fully third-order ordinary differential equations. Comput. Math. Appl. 59 3464-3471 (2010)

12. Nieto, J.: Nonlinear second-order periodic boundary value problems. J. Math. Anal. Appl. 130, $22-29$ (1988)

13. Prasad, K.R., Sreedhar, N., Wesen, L.T.: Existence of positive solutions for higher order $p$-Laplacian boundary value problems. Mediterr. J. Math. 15, 19 (2018)

14. Torres, P.: Existence of one-signed periodic solution of some second-order differential equations via a Krasnoselskii fixed point theorem. J. Differ. Equ. 190, 643-662 (2003)

\section{Submit your manuscript to a SpringerOpen ${ }^{\circ}$} journal and benefit from:

- Convenient online submission

- Rigorous peer review

Open access: articles freely available online

- High visibility within the field

- Retaining the copyright to your article 